\title{
Correspondence
}

\section{Channel Impulse Response Tap Prediction for Time-Varying Wireless Channels}

\author{
J. Akhtman and L. Hanzo
}

\begin{abstract}
In this paper, we perform a comparative study of both the achievable performance and the associated computational complexity of two major time-domain prediction strategies proposed for employment in wireless mobile communication systems. Specifically, we investigate the intrinsic design tradeoffs of the so-called stationary robust predictor and the adaptive Recursive-Least-Squares (RLS) predictor. We demonstrate that the RLS predictor outperforms its robust counterpart at a cost of slightly higher computational complexity, and hence, the RLS predictor constitutes a better alternative for employment in wireless transceivers.
\end{abstract}

Index Terms-Channel prediction of time-varying channels, minimum mean square error (MMSE) channel prediction, orthogonal-frequencydivision-multiplexing (OFDM), recursive least squares.

\section{INTRODUCTION}

The channel impulse response (CIR) predictor predicts the evolution of each CIR tap in the time domain (TD) and constitutes an important ingredient of many advanced wireless mobile transceivers, such as, for instance, the decision-directed channel estimation (DDCE)-aided [1] orthogonal-frequency-division-multiplexing (OFDM) scheme discussed in detail in [2]. Two major CIR tap prediction strategies have been proposed and discussed in the context of DDCE-aided OFDM systems. Specifically, the so-called robust implementation of the stationary minimum mean square error (MMSE) CIR predictor was introduced in [3] and further elaborated upon in [2]. The robust CIR predictor considered in [3] assumes a constant-valued limitedsupport channel scattering function [2] during the design of the CIR tap prediction filter and, hence, relies on the assumption of encountering the worst possible channel conditions. It was shown in [2] that this is the worst-case performance, regardless of the actual channel conditions encountered, provided that the scattering function encountered does not exceed its assumed bandwidth [2, Sec. 14.6.2]. On the other hand, the adaptive recursive-least-squares (RLS) method of CIR prediction was explored in detail in [4]. As opposed to the robust CIR predictor in [3], the RLS CIR predictor does not require any explicit information concerning the channel conditions encountered. Our aim is to characterize the achievable performance of both methods considered and draw conclusions concerning their relative merits.

Manuscript received August 18, 2005; revised May 20, 2006 and January 10, 2007. This work has formed part of the Wireless Enabling Techniques work area of the Core 3 Research Programme and was supported in part by the Virtual Centre of Excellence in Mobile and Personal Communications, Mobile VCE, www.mobilevce.com, and in part by the Engineering and Physical Sciences Research Council. Fully detailed technical reports on this research are available to Industrial Members of Mobile VCE. The review of this paper was coordinated by Prof. H. Leib.

The authors are with the School of Electronics and Computer Science, University of Southampton, SO171BJ Southampton, U.K. (e-mail: lh@ecs. soton.ac.uk).

Digital Object Identifier 10.1109/TVT.2007.900395

\section{TD A Priori CIR PREDICTION}

The aim of the a priori CIR predictor within the DDCE-OFDM receiver [2], [3] is to predict the CIR taps $\left\{\alpha_{1}[n+1], \ldots, \alpha_{L}[n+\right.$ $1]\}$ associated with the future OFDM symbol instances, given the previous CIR taps, namely, the a posteriori CIR tap estimates $\left\{\left\{\hat{\alpha}_{l}[n]\right\},\left\{\hat{\alpha}_{l}[n-1]\right\}, \ldots\right\}$. As outlined in [2], the $l$ th CIR component $\alpha_{l}[n]$ undergoes a narrowband fading process characterized by associated cross-correlational function, which can be described as follows:

$$
E\left\{\alpha_{l}^{*}[n] \alpha_{l^{\prime}}[n-m]\right\}=\sigma_{l}^{2} r_{\mathrm{t}}[n, m] \delta\left[l-l^{\prime}\right]
$$

where $\sigma_{l}^{2}$ is the magnitude of the corresponding power-delay-profile component [2], and $r_{t}[n, m]$ denotes the TD autocorrelation function, while $\delta[\cdot]$ is the Kronecker delta function. Consequently, the narrowband fading process characterized by (1) may be approximately modeled as an autoregressive process of the order of $N_{\text {prd }}$ [2], and the a priori estimate $\check{\alpha}_{l}[n+1]$ may be obtained according to

$$
\check{\alpha}_{l}[n+1]=\boldsymbol{q}_{l}^{\mathrm{H}}[n] \hat{\boldsymbol{\alpha}}_{l}[n], \quad l=1, \ldots, L
$$

where $\boldsymbol{q}_{l}[n]=\left(q_{l}[0], \ldots, q_{l}\left[N_{\text {prd }}-1\right]\right)^{\mathrm{T}}$ denotes the $l$ th CIR tap's finite-impulse-response prediction filter coefficient vector, and we define $\hat{\boldsymbol{\alpha}}_{l}[n]=\left(\hat{\alpha}_{l}[n], \hat{\alpha}_{l}[n-1], \ldots, \hat{\alpha}_{l}\left[n-N_{\text {prd }}+1\right]\right)^{\mathrm{T}}$.

\section{MMSE CIR PREDICTION}

Following the MMSE approach [2], [3], the aforementioned fading process is assumed to be wide sense stationary (WSS). Consequently, the corresponding time-invariant prediction filter coefficient vector $\boldsymbol{q}_{l}$ of (2) is attained by solving a set of linear equations, which may be obtained upon left multiplying the complex conjugates of both sides of (2) with the column vector $\boldsymbol{\alpha}_{l}[n]$ and subsequently obtaining the expectation value over the TD index $n$ as follows:

$$
E\left\{\boldsymbol{\alpha}_{l}[n] \alpha_{l}^{*}[n+1]\right\}=E\left\{\boldsymbol{\alpha}_{l}[n] \boldsymbol{\alpha}_{l}^{\mathrm{H}}[n]\right\} \boldsymbol{q} .
$$

Thus, we arrive at the set of Yule-Walker equations, which may be expressed as [5]

$$
\boldsymbol{r}_{\mathrm{t}}=\boldsymbol{R}_{\mathrm{apt} ; l} \boldsymbol{q}_{l}
$$

where

$$
\boldsymbol{r}_{\mathrm{t}}=\frac{1}{\sigma_{l}^{2}} E\left\{\boldsymbol{\alpha}_{l}[n] \alpha_{l}^{*}[n+1]\right\}
$$

and

$$
\boldsymbol{R}_{\mathrm{apt} ; l}=\frac{1}{\sigma_{l}^{2}} E\left\{\hat{\boldsymbol{\alpha}}_{l}[n] \hat{\boldsymbol{\alpha}}_{l}^{\mathrm{H}}[n]\right\}=\boldsymbol{r}_{\mathrm{t}} \boldsymbol{r}_{\mathrm{t}}^{\mathrm{H}}+\frac{\sigma_{w}^{2}}{\sigma_{l}^{2}} \boldsymbol{I}
$$

are the autocorrelation vector and the $\left(N_{\text {prd }} \times N_{\text {prd }}\right)$-dimensional a posteriori sample covariance matrix of the CIR taps [2], respectively, while $\sigma_{w}^{2}$ and $\sigma_{l}^{2}$ are the noise variance and the average power of the lth CIR tap. Consequently, the solution of (4) is given by

$$
\boldsymbol{q}_{l}=\boldsymbol{R}_{\mathrm{apt} ; l}^{-1} \boldsymbol{r}_{\mathrm{t}} .
$$

A particularly popular model of describing the underlying channel statistics is constituted by Jakes' model [6], where the autocorrelation 
vector $\boldsymbol{r}_{\mathrm{t}}$ may be formulated as $r_{\mathrm{t}}[m]=r_{J}[m]=J_{0}\left(2 \pi f_{\mathrm{D}} m\right), m=$ $1,2, \ldots, N_{\text {prd }}$, and $J_{0}(x)$ is a zero-order Bessel function of the first kind. The corresponding a posteriori covariance matrix $\boldsymbol{R}_{\text {apr }}$ and the resultant CIR predictor's filter coefficients are given by (6) and (7), respectively. Finally, the prediction is performed according to (2).

\section{RoBUST CIR PREDICTION}

The aforementioned MMSE CIR-tap prediction process exhibits a relatively high accuracy under the assumption of having a perfect knowledge of the channel statistics, namely, the autocorrelation function $\boldsymbol{r}_{\mathrm{t}}$, as well as the noise variance $\sigma_{w}^{2}$. However, it suffers from a substantial performance degradation, when the actual channel statistics encountered deviate from the model assumed. The potential statistical mismatch becomes increasingly detrimental in wireless mobile environments, where the channel conditions and the corresponding statistics are time dependent, and hence, the fading process cannot be assumed to be WSS. Nevertheless, as suggested in [3], the CIR tap prediction accuracy may be maintained even in mismatched channel conditions, if the appropriate worst-case assumptions concerning the channel statistics are made. More specifically, the MSE exhibited by the mmse CIR predictor of (2) is upperbounded by the MSE encountered, when communicating over an ideally band-limited channel having a perfect low-pass Doppler power spectral density [3]. The corresponding sinc-shaped a priori autocorrelation vector may be expressed as

$$
r_{\mathrm{t} ; \mathrm{rob}}[m]=\frac{\sin 2 \pi f_{\mathrm{D}} m}{2 \pi f_{\mathrm{D}} m}, \quad m=1,2, \ldots, N_{\mathrm{prd}} .
$$

Consequently, we arrive at the concept of designing the so-called robust mmse predictor [2], [3] of Li et al. [3], which assumes encountering the worst possible constant-valued support-limited channel scattering function yielding the highest possible mmse. The corresponding channel statistics are determined by two parameters, namely, the assumed model's maximum Doppler frequency $f_{\mathrm{D} ; \mathrm{prd}}$, as well as the assumed AWGN variance $\rho$. It was demonstrated in [3] that regardless of the CIR's shape, no CIR tap estimation mse performance degradation occurs, as long as the actual Doppler frequency $f_{\mathrm{D}}$ does not exceed the Doppler frequency $f_{\mathrm{D} ; \text { prd }}$ assumed. Furthermore, the resultant CIR predictor exhibits robustness to the mismatch of the AWGN variance in comparison to the value assumed.

\section{AdAPTIVE RLS CIR PREDICTION}

On the other hand, in the RLS-based adaptive CIR tap prediction approach in [4] and [7], no assumptions were made concerning the channel's stationarity. Consequently, the time-variant lth CIR tap's predictor filter coefficient vector $\boldsymbol{q}_{l}[n]$ is calculated by minimizing the following scalar cost function:

$$
J_{\mathrm{RLS} ; l}[n]=\sum_{i=1}^{n} \beta^{n-i}\left|\alpha_{l}[i+1]-\boldsymbol{q}_{l}^{\mathrm{H}}[n] \boldsymbol{\alpha}_{l}[i]\right|^{2}
$$

where $\beta \in(0,1)$ is the so-called forgetting factor [7], which accounts for possible deviations of the fading process encountered from the WSS assumption. The resultant recursive update for $\boldsymbol{q}_{l}[n]$ is given by

$$
\boldsymbol{q}_{l}[n]=\boldsymbol{q}_{l}[n-1]+\boldsymbol{k}_{l}[n-1] e_{l}^{*}[n]
$$

where

$$
e_{l}[n]=\hat{\alpha}_{l}[n]-\boldsymbol{q}_{l}^{\mathrm{H}}[n-1] \hat{\boldsymbol{\alpha}}_{l}[n-1]
$$

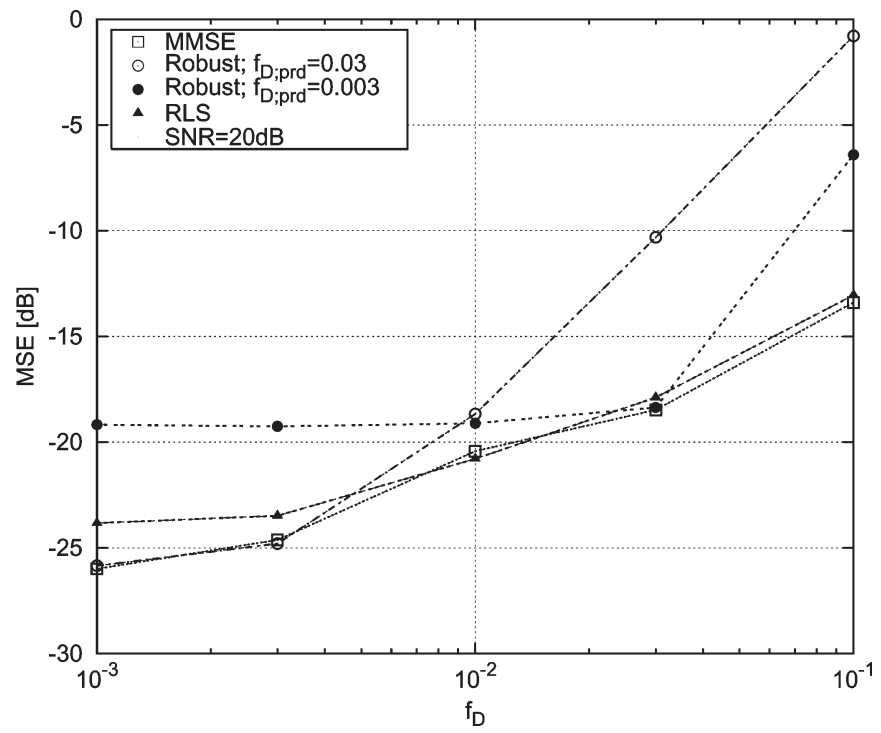

Fig. 1. Mean square error exhibited by the MMSE, robust, and RLS a priori CIR predictors as a function of the OFDM-symbol-normalized Doppler frequency encountered. Two cases of robust prediction are considered, namely, when $f_{\mathrm{D} ; \operatorname{prd}}=0.03$ and $f_{\mathrm{D} ; \mathrm{prd}}=0.003$. The results correspond to an SNR of $20 \mathrm{~dB}$.

is the prediction error, while [7]

$$
\boldsymbol{k}_{l}[n]=\frac{\boldsymbol{P}_{l}[n-1] \hat{\boldsymbol{\alpha}}_{l}[n]}{\boldsymbol{\beta}+\hat{\boldsymbol{\alpha}}_{l}^{\mathrm{H}}[n] \boldsymbol{P}_{l}[n-1]_{l} \hat{\boldsymbol{\alpha}}_{l}[n]}
$$

denotes the RLS gain vector. Furthermore, the matrix $\boldsymbol{P}_{l}[n]$ is the inverse of the $l$ th CIR tap's $\left(N_{\text {prd }} \times N_{\text {prd }}\right)$-dimensional sample covariance matrix, which can be recursively calculated as follows:

$$
\boldsymbol{P}_{l}[n]=\frac{1}{\beta}\left(\boldsymbol{I}-\boldsymbol{k}_{l}[n] \hat{\boldsymbol{\alpha}}_{l}^{\mathrm{H}}[n]\right) \boldsymbol{P}_{l}[n-1] .
$$

As pointed out in [8], the choice of the forgetting factor's value $\beta$ has only a moderate effect on the performance of the resultant predictor. Specifically, in our investigations, we used the value suggested in [8], namely, $\beta=0.99$.

\section{Simulation Results}

In our simulations, we employed an OFDM system having 128 QPSK-modulated orthogonal subcarriers. Furthermore, we assumed a block-fading multipath channel characterized by the Jakes fading model [6], as well as the COST-207 BU multipath model [9].

Fig. 1 illustrates the achievable mse performance of the CIR prediction methods considered as a function of the Doppler frequency $f_{\mathrm{D}}$ encountered. It can be seen that the MMSE CIR predictor, which relies on a perfect a priori knowledge of the underlying channel statistics, represents the best-case MMSE performance achievable by a linear predictor. Furthermore, the robust CIR predictor exhibits a relatively high performance, i.e., low mse, as long as the actual Doppler frequency encountered does not exceed that of the assumed. Finally, the RLS CIR predictor represented by triangles in Fig. 1, which does not require any explicit knowledge concerning the channel statistics, exhibits a nearoptimum performance over the entire range of the values of $f_{\mathrm{D}}$.

Furthermore, Fig. 2 illustrates the achievable mse performance of the CIR prediction methods considered as a function of the SNR encountered. Once again, the mmse CIR predictor exhibits the highest achievable performance, leading to the lowest MSE. The robust CIR predictor exhibits a relatively high performance, as long as the SNR encountered does not exceed the value $1 / \rho$ assumed. On the other 


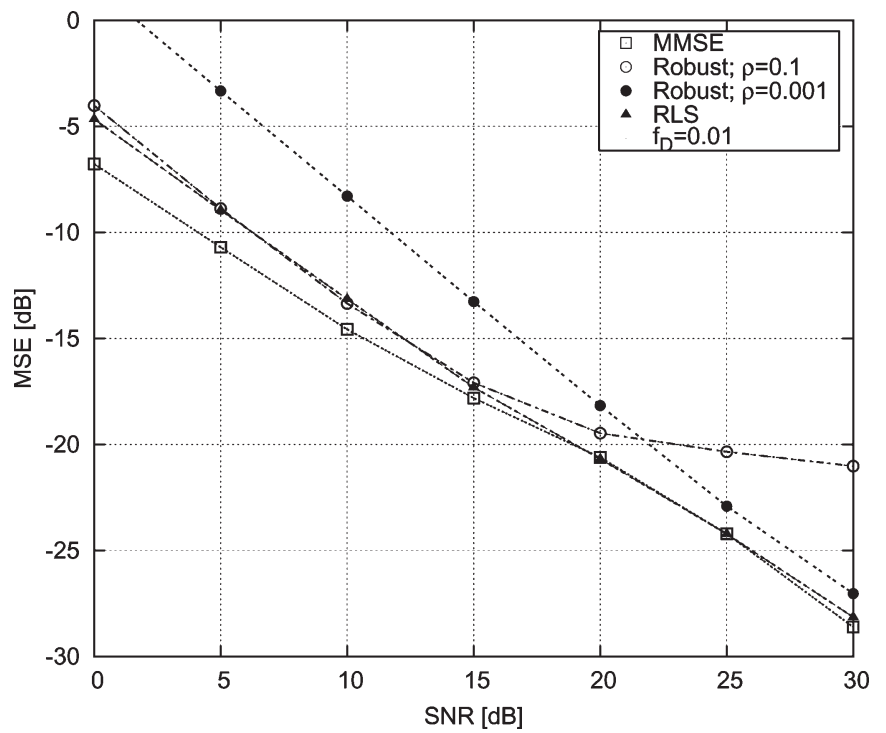

Fig. 2. Mean square error exhibited by the MMSE, robust, and RLS a priori CIR predictors as a function of the SNR encountered. Two cases of robust prediction are considered, namely, when $\rho=0.1$ and $\rho=0.001$. The results correspond to the symbol-rate-normalized Doppler frequencies of $f_{\mathrm{D}}=0.01$

hand, the RLS predictor exhibits near optimum performance over the whole range of SNR values.

Additionally, the order of the computational complexity associated with both CIR predictors considered in the context of a DDCE-OFDM system and quantified in terms of the total number of complex multiplications and additions per OFDM symbol may be expressed as $O\left(K \log _{2} K+L N_{\text {prd }}\right)$ and $O\left(K \log _{2} K+L N_{\text {prd }}+L N_{\text {prd }}^{2}\right)$ for the robust [2] and RLS [4] CIR predictors, respectively. ${ }^{1}$ Explicitly, the order of complexity imposed by the RLS CIR predictor is only slightly higher than that associated with the robust CIR predictor.

\section{CONCLUSION}

We have demonstrated that the adaptive RLS TD prediction scheme outperforms the stationary robust CIR tap predictor and, therefore, constitutes a better design alternative for employment in wireless mobile transceivers utilizing a DDCE-aided OFDM modulation scheme. To elaborate further, both methods have to operate in the absence of reliable a priori information regarding the channel conditions. Therefore, the availability of reliable estimates of the parameters $f_{\mathrm{D}}$ and $\rho$ may not be assumed. In this light, our results demonstrate that the adaptive RLS prediction method outperforms its robust counterpart in the scenario of having poor estimates of the parameters $f_{\mathrm{D}}$ and $\rho$.

\section{REFERENCES}

[1] M. Munster and L. Hanzo, "Parallel interference cancellation assisted decision-directed channel estimation for OFDM systems using multiple transmit antennas," IEEE Trans. Wireless Commun., vol. 4, no. 5, pp. 2148-2162, Sep. 2005.

[2] L. Hanzo, M. Münster, B. Choi, and T. Keller, OFDM and MC-CDMA for Broadband Multi-User Communications, WLANs and Broadcasting. Hoboken, NJ: Wiley, 2003. 992 p.

${ }^{1} \mathrm{~K}$ denotes the number of subcarriers comprising the OFDM symbol, while $L$ is the number of nonzero CIR taps encountered.
[3] Y. Li, L. Cimini, and N. Sollenberger, "Robust channel estimation for OFDM systems with rapid dispersive fading channels," IEEE Trans. Commun., vol. 46, no. 7, pp. 902-915, Apr. 1998.

[4] D. Schafhuber and G. Matz, "MMSE and adaptive prediction of timevarying channels for OFDM systems," IEEE Trans. Wireless Commun., vol. 4, no. 2, pp. 593-602, Mar. 2005.

[5] S. M. Kay, Modern Spectral Estimation: Theory and Application. Englewood Cliffs, NJ: Prentice-Hall, 1988.

[6] W. Jakes, Jr., Ed., Microwave Mobile Communications. New York: Wiley, 1974.

[7] S. Haykin, Adaptive Filter Theory. Englewood Cliffs, NJ: Prentice-Hall, 1996.

[8] D. Schafhuber, G. Matz, and F. Hlawatsch, "Adaptive prediction of time-varying channels for coded OFDM systems," in Proc. IEEE Int. Conf. Acoust., Speech, Signal Process., May 2002, vol. 3, pp. III-2549III-2552.

[9] M. Failli, Digital Land Mobile Radio Communications COST 207, 1989, Eur. Comm. Tech. Rep.

\section{Joint Channel and Carrier Offset Estimation for Synchronous Uplink CDMA Systems}

Samir Attallah, L. B. Thiagarajan, Hongyi Fu, and Ying-Chang Liang

\begin{abstract}
This paper addresses the problem on blindly estimating the channel impulse response (CIR) and the carrier frequency offset (CFO) in the uplink transmissions of multiuser code division multiple access system. Two blind subspace-based CIR and CFO estimation methods, namely, the exact determinant minimization method and the approximate determinant minimization method, are proposed. The performance of the proposed methods is compared with the available solution based on the generalized eigenvalue problem method (GEVPM). The computational complexity of the proposed methods is compared with that of GEVPM and the estimator proposed by $\mathrm{Li}$ and Liu. Simulation results are given to show that the proposed methods give better performance than the GEVPM and have a wide CFO acquisition range.
\end{abstract}

Index Terms-Carrier offset, channel estimation, code division multiple access, noise subspace.

\section{INTRODUCTION}

Direct sequence code division multiple access (DS-CDMA) is one of the popular multiple-access techniques in many wireless applications because of its improved capacity [1], simultaneous spectrum sharing capability, and inherent resistance to jamming, interception, and multipath propagation effects [2]. The above advantages of a CDMA technique have led CDMA to be used in 3G standards [3], [4]. In the uplink scenario, the transmitted signal from each user propagates through a multipath channel, which distorts the orthogonality between the spreading codes of the users. Therefore, to have a reliable communication, this multipath channel must be equalized at the receiver [5], [6]. Besides the multipath fading, the received signal also has a small carrier frequency offset (CFO) due to Doppler shift and a possible carrier frequency mismatch between the transmit and receive oscillators.

Manuscript received October 28, 2005; revised July 19, 2006 and October 20, 2006. The review of this paper was coordinated by Dr. M. Stojanovic.

S. Attallah and L. B. Thiagarajan are with the Department of Electrical and Computer Engineering, National University of Singapore, Singapore 117576 (e-mail: attallah@ieee.org; lb_thiagarajan@ieee.org).

H. Fu and Y.-C. Liang are with the Institute for Infocomm Research, Singapore 119613 (e-mail: fuhy@i2r.a-star.edu.sg; ycliang@i2r.a-star.edu.sg).

Digital Object Identifier 10.1109/TVT.2007.898392 